\title{
The Role of Pain Scoring in Multi-detector Computed Tomography Indications in Pediatric Occult Fractures
}

\author{
Hüseyin Mutlu ${ }^{1}$, Ahmet Çağlar ${ }^{1 *}$, Ekrem Taha Sert ${ }^{1}$
}

\author{
${ }^{1}$ Aksaray University Medical School, \\ Department of Emergency Medicine, \\ Aksaray, Turkey

\section{*Correspondence} \\ drcaglar4@gmail.com (Ahmet Çağlar)
}

\begin{abstract}
Introduction: Computed tomography (CT) is used in emergency orthopedic trauma cases with suspicion of fracture that cannot be detected on X-ray. Also pain during examination in patients with fracture is important for clinicians. We aimed to investigate whether pain severity is indicative of multi-detector CT (MDCT). Methods: A total of 175 patients included in the study. Visual analogue scale (VAS) was used to evaluate pre-examination pain in all patients. The most painful and suitable region for palpation were determined, palpated and the patients were asked to re-score their pain. In this way, the local VAS score was determined. MDCT was performed in all patients with general VAS of $>5$. MDCT results were compared with the VAS scores. Results: Fracture detection in MDCT was $61.1 \%$ in patients with general VAS score of $\geq 5$. Of the 105 patients with the local VAS score of $\geq 7,90$ had fractures. In these patients, the sensitivity, specificity, positive predictive value and negative predictive value of MDCT for detecting fractures were $84.1 \%, 77.9 \%, 85.7 \%$ and $75.7 \%$, respectively. Conclusion: We believe that MDCT should be performed for suspected fracture if pain severity is high in isolated extremity trauma cases without fracture detection on X-ray.
\end{abstract}

\section{Keywords}

Multi-detector Computed tomography, Pediatric trauma, Pain, Visual analogue scale

\section{Introduction}

Childhood orthopedic trauma is one of the most predominant reasons for admission to Emergency Department (ED). Approximately $9 \%$ of children admitted to ED for trauma have fractures [1]. It is estimated that approximately half of the children have had bone fracture at least once during childhood [2]. Fractures in children are particularly important because a child's bone structure is different from an adult's bone structure. Pediatric fractures are difficult to diagnose as their radiological appearance is highly variable.

Early diagnosis is important in patients admitted to ED because of the limited time frame for a successful treatment. Accurate imaging plays an important role in achieving this goal. Direct X-rays have been used for a long time as the primary imaging modality for evaluating the musculoskeletal system. Direct X-rays are one of the most frequently used examination technique in ED despite the availability of many new-generation imaging modalities
[3]. In ED, computed tomography (CT) is used as an adjunct modality to $\mathrm{X}$-rays while imaging bone structures with complex anatomy when direct X-rays fail to detect fractures [4]. Occult fractures occur after trauma and cannot be detected using direct X-rays; they are characterized by pain and detected using multi-detector CT (MDCT) [5]. Early diagnosis of occult fractures is crucial for understanding the patient's symptoms and preventing complications. Advanced imaging tools, such as CT, are highly valuable in this context. However, currently there is a lack of a widely accepted evidence-based policy regarding the use of $\mathrm{CT}$ for assessing pediatric fractures.

It is very important for emergency physicians to describe and effectively manage pain when a patient is admitted to ED. The Visual Analogue Scale (VAS) has been used for measuring pain since the 1920s [6]. VAS is a valid and reliable measure of both chronic and acute pain intensity [7-10]. VAS is suitable for use in ED settings and can easily 


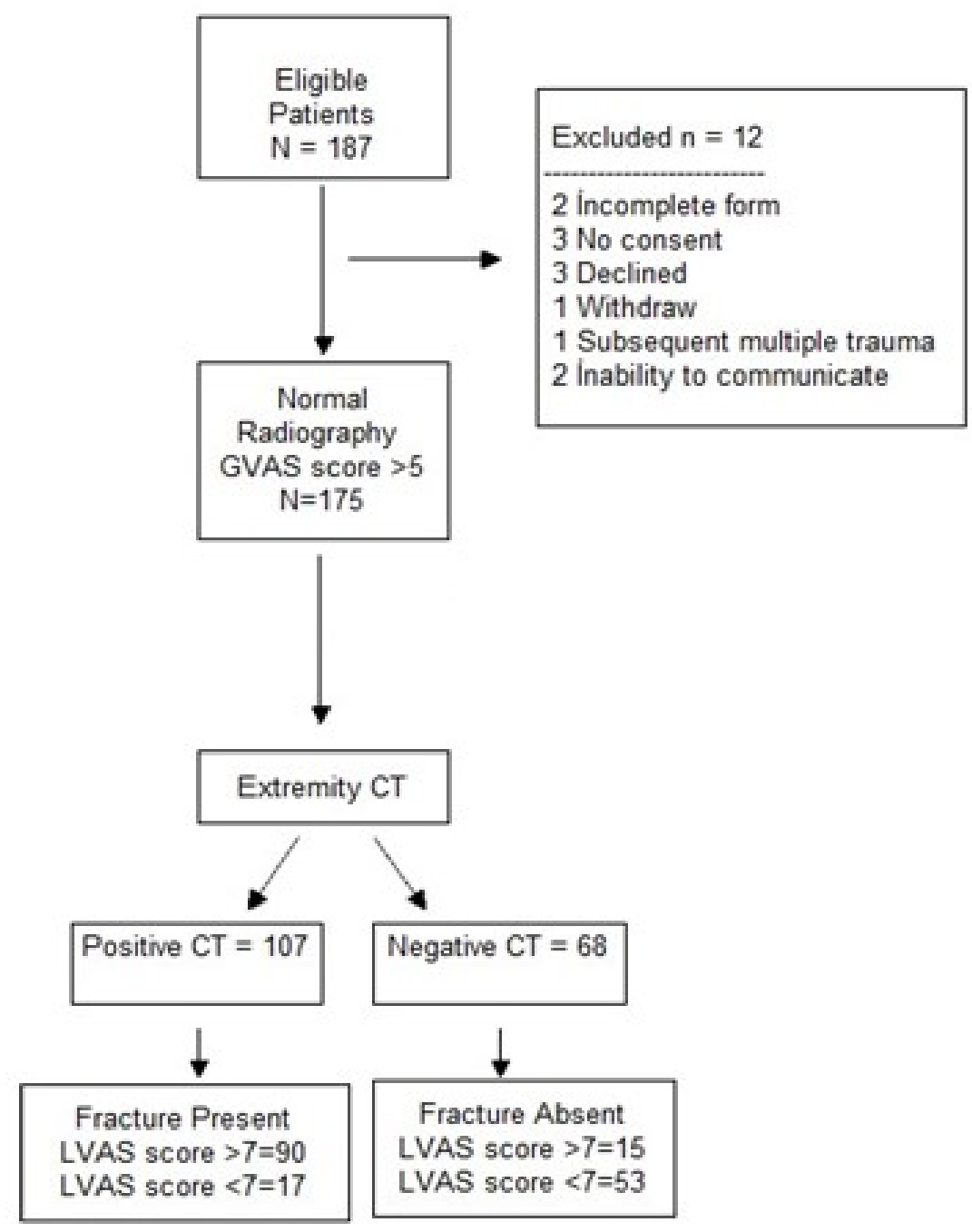

F I G U R E 1. Flowchart of the study. VAS: Visual Analogue Scale, G-VAS: General VAS, L-VAS: Local VAS, MDCT: Multi-detector computer tomography.

\section{TA B L E 1. General characteristics of study population.}

Number of patients

Age, y, median (IQR)

Male sex, n, (\%)

Positive CT findings, $\mathrm{n},(\%)$

$93(53.1)$

$107(61.1)$

Upper extremity positive MDCT findings, $n,(\%) \quad 68$ (63.6)

Lower extremity positive MDCT findings, n, (\%) 39 (36.4)

General VAS score, median (IQR)

$6(5-7)$

Local VAS score, median (IQR)

VAS: Visual Analogue Scale, MDCT: Multi-detector computer tomography.

be applied to children aged 8-16 years [9]. In VAS, a score of 0 indicates "no pain at all," and a score of 10 indicates "worst pain imaginable" [10].

In the present study, we aimed to investigate pain severity and fracture detection rates among pediatric patients using MDCT as well as elucidate whether pain severity was indicative of MDCT.

\section{Materials and Methods}

\subsection{Study population}

Patients aged 8-16 years who had been admitted to Aksaray University Training and Research Hospital Emergency Department between June 2018 and October 2019 owing to isolated extremity trauma and in whom we had failed to detect fractures on X-ray scans were prospectively evaluated.

The suitability of X-ray was gauged before evaluating the pathological findings on X-ray scans. Non-diagnostic Xrays were excluded due to poor image quality and motion artefacts. Furthermore, patients aged $<8$ years or $>16$ years, patients with open wounds or disorders such as malunion, patients with mental incapacity for VAS evaluation, patients with a VAS score of $<5$, patients with no history of trauma, patients with multiple trauma (simultaneous 
TA B L E 2. Comparison between patients who had positive MDCT findings and those who not.

\begin{tabular}{|c|c|c|c|}
\hline & positive MDCT findings $(n=107)$ & negative MDCT findings $(n=68)$ & p Value \\
\hline Age, y, median (IQR) & $12(9-14)$ & $11(8-13)$ & 0.11 \\
\hline Sex, male, n (\%) & $56(60.2)$ & $37(39.8)$ & 0.79 \\
\hline G-VAS score, median (IQR) & $7(6-7)$ & $5(5-6)$ & $<0.001$ \\
\hline L-VAS score, median (IQR) & $8(7-9)$ & $6(6-6)$ & $<0.001$ \\
\hline L-VAS Group & & & $<0.001$ \\
\hline L-VAS score: $5-6, \mathrm{n}(\%)$ & $17(15.9)$ & $53(77.9)$ & \\
\hline L-VAS score: $7-10, \mathrm{n}(\%)$ & $90(84.1)$ & $15(22.1)$ & \\
\hline
\end{tabular}

VAS: Visual Analogue Scale, G-VAS: General VAS, L-VAS: Local VAS, MDCT: Multi-detector computer tomography.

TA B L E 3. VAS scores of patients with positive MDCT findings according to anatomical location.

\begin{tabular}{|c|c|c|c|c|}
\hline & $\mathrm{n},(\%)$ & $\begin{array}{l}\text { GVAS score Median } \\
\text { (IQR) }\end{array}$ & $\begin{array}{c}\text { L-VAS score Median } \\
\text { (IQR) }\end{array}$ & p value \\
\hline Radial head fracture (intraarticular) & $6(5.6)$ & $5.5(5-6)$ & $6.5(6-7.5)$ & 0.066 \\
\hline Olecranon fracture & $5(4.7)$ & $6(5.5-7)$ & $8(7-8)$ & 0.063 \\
\hline Distal radius fracture & $19(17.7)$ & $7(6-8)$ & $8(7-9)$ & $<0.001$ \\
\hline Scaphoid fracture & $18(16.8)$ & $7(7-8)$ & $9.5(9-10)$ & $<0.001$ \\
\hline Pisiform, capitate, trapezium fracture & $13(12.1)$ & $6(5-6)$ & $7(6-7)$ & 0.001 \\
\hline Lunate, unlar styloid, proximal metacarpal fractures & $7(6.5)$ & $5(5-6)$ & $7(6-7)$ & 0.011 \\
\hline SH-I distal fibula fractures & $7(6.5)$ & $6(6-7)$ & $7(6-8)$ & 0.034 \\
\hline Tibial plateau fracture & $9(8.4)$ & $7(5.5-7.5)$ & $9(8-9.5)$ & 0.005 \\
\hline Talus fracture & $13(12.1)$ & $7(7-8)$ & $9(8-9)$ & 0.007 \\
\hline Calcaneus fracture & $10(9.3)$ & $7(7-8.3)$ & $9(7.8-9.3)$ & 0.023 \\
\hline
\end{tabular}

VAS: Visual Analogue Scale, G-VAS: General VAS, L-VAS: Local VAS, MDCT: Multi-detector computer tomography, SH-I:Salter-Harris Type I.

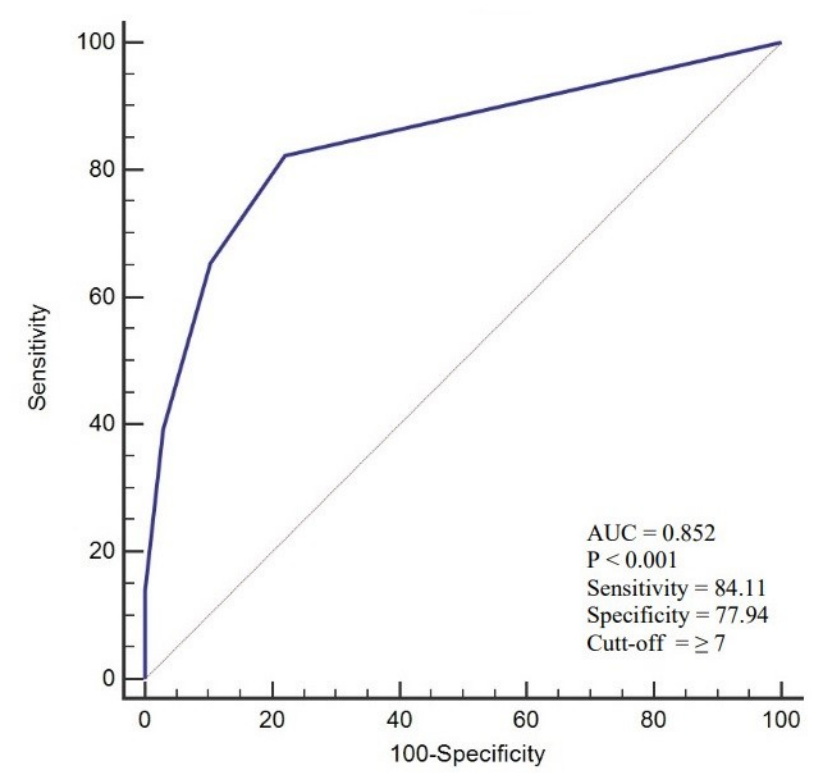

F IGURE 2. ROC curve analysis of Local Visual Analogue Scale.

injury at two or more body sites), and patients who took medication for pain before admission to ED were excluded
TA B L E 4. Performance parameters of L-VAS in predicting occult fractures.

\begin{tabular}{lcc} 
Performance parameters & & $\begin{array}{c}\text { 95\% confidince } \\
\text { interval }\end{array}$ \\
\hline Sensitivity & 84.11 & $75.78-90.46$ \\
Specificity & 77.94 & $66.24-87.1$ \\
\hline Positive predictive value & 85.71 & $79.21-90.43$ \\
Negative predictive value & 75.71 & $66.44-83.08$ \\
\hline Positive likelihood ratio & 3.81 & $2.42-6$ \\
\hline Negative likelihood ratio & 0.2 & $0.13-0.31$ \\
\hline Area under curve & 0.85 & $0.781-0.894$ \\
\hline P value & $<0.001$ & \\
\hline
\end{tabular}

L-VAS: Local Visual Analogue Scale.

from the study.

\subsection{Study design and setting}

The study was conducted in compliance with the Declaration of Helsinki and approved by Aksaray University School of Medicine, Aksaray Education and Research Hospital Scientific Research Evaluation 
Committee (2018/211).

The patients were examined by an emergency medicine specialist after admission to ED. Before the examination, all patients were asked to rate the level of pain between 0 and 10 , which was recorded as the general VAS (G-VAS) score. If the G-VAS score was $\geq 5$, the patients were asked to specify the point at which the pain was the most severe, and that point was then palpated using the inside of the thumb. The patients were asked to re-score their pain using VAS, and this re-scored VAS was recorded as local VAS (L-VAS). After examination, pain medication was administered to the patients if needed.

Standard anterior-posterior (AP) as well as standard lateral and oblique X-ray scans were taken for all patients. $\mathrm{X}$-ray scans were examined by two emergency medicine physicians using the hospital's computer system (Probel Hospital Information Management System, PACS).

If the findings from the X-ray scans are normal according to both the doctors; the parents of the patients were informed that MDCT would be used as a diagnostic imaging method to detect possible bone damage and the treatment strategy would be re-planned. A detailed written informed consent was also obtained from the parents or guardians of all patients for participating in this study. The same two specialists performed MDCT on the patients included in this study and evaluated its results. Patients were treated according to the MDCT results.

The same flow chart was applied for all the patients included in the study (Fig. 1).

\subsection{Data analysis}

Data were analyzed using Statistical package social sciences (SPSS, version 15.0 Inc., Chicago IL, ABD). Descriptive statistical methods (percentage, median, mean, and standard deviation) were used to evaluate the data. Data distribution was evaluated using the Kolmogorov-Smirnov test. Chi-square test and Student's t-test were used for comparing categorical and continuous variables between groups, respectively. Mann-Whitney U-test or KruskalWallis variance analysis was used for non-normally distributed continuous and categorical variables. The diagnostic characteristics of L-VAS in terms of predicting fracture on MDCT were analyzed using receiver operating characteristics (ROC) curve. The cut-off value was determined using the Youden index. ROC curves were evaluated using Med Calc Software Version 19.1. p value of $<0.05$ was considered to be statistically significant.

\section{Results}

A total of 187 patients were found to be eligible for this study, and 12 of those patients were excluded because of the reasons depicted in the flow chart (Fig. 1); thus, 175 patients were finally included.

A total of 175 patients aged between 8 and 16 years who were admitted to ED owing to isolated extremity trauma with the G-VAS score of $\geq 5$ and whose fractures could not be detected on X-ray scans were included in the present study; 93 of the patients were males and 82 were females (53.1\% and $46.8 \%$, respectively). The median age of the patients was $11(9-14)$ years. The median G-VAS score at the time of admission was $6(5-7)$ and the median LVAS score at the time of examination was 7 (6-9). The general characteristics of the patients included in the study are summarized in Table 1.

Patients were divided into the following two groups: LVAS (5-6) and L-VAS (7-10). The distribution of patients with and without fractures detected on MDCT in the abovementioned groups is summarized in Table 2. When the localization of bone fracture was examined, it was found that occult fracture of the upper extremity was the most common $(63.6 \%, \mathrm{n}: 68)$. Distal radius fractures were the most frequently occurring occult fractures in the upper extremity (17.7\%, n:19), and talus fractures were the most frequently occurring occult fractures in the lower extremity $(12.1 \%, \mathrm{n}: 13)$. When fractures were evaluated according to localization, the highest L-VAS score was obtained for scaphoid fractures (9-10; median, 9.5) and the lowest GVAS was obtained for lunate, ulnar, styloid, and proximal metacarpal fractures (5-6; median, 5). VAS score and comparisons based on anatomical localization are presented in Table 3.

All patients with a G-VAS score of $\geq 5$ underwent MDCT. While 107 (61.1\%) patients had fractures, 68 (38.9\%) patients did not. The cut-off value obtained using the ROC curve for L-VAS was $\geq 7$ (sensitivity: $84.11 \%$; specificity: $77.94 \%$; area under the curve(AUC): 0.852 ; 95\% confdence interval: $0.781-0.894 ; \mathrm{p}<0.001$ ) (Fig. 2). The performance characteristics of L-VAS are shown in Table 4. A total of 105 patients $(60 \%)$ had a L-VAS score of $\geq 7$. No fractures were detected in $15(14.3 \%)$ of these patients. While 22 of 90 patients had significant fractures requiring further treatment, 68 patients had insignificant fractures.

\section{Discussion}

It is difficult to detect occult fractures in children with orthopedic trauma. MDCT is a highly useful diagnostic tool, especially in cases where direct X-ray is inconclusive and findings are suspicious [11]. In the present study, we evaluated extremity trauma patients without fracture via Xray scans and concluded that patients with a high VAS score should be evaluated for a possible fracture using MDCT. MDCT revealed fractures in patients who had normal X-ray findings and demonstrated that the inadequacy of X-rays for detecting some crucial extremity fractures. We found that occult fracture detection rate was $61.1 \%$ in all patients with a G-VAS score of $\geq 5$ (n:175). Only 15 (14.3\%) of the patients with an L-VAS score of $\geq 7$ had no fractures. In patients with an L-VAS score of $\geq 7$, the sensitivity, specificity, PPV, and NPV of MDCT for fracture detection were $84.11 \%, 77.94 \%, 85.71 \%$, and $75.71 \%$, respectively. We recommended that patients with a G-VAS score of $\geq$ 5 and an L-VAS score of $\geq 7$ but showing no fractures 
on X-ray scans should be evaluated using MDCT. In the present study, we demonstrated that occult fractures can be identified more safely using MDCT; thus, the treatment can be substantially modified.

Although direct X-ray is accepted as the primary imaging modality in skeletal trauma, MDCT has become an important adjunct imaging method especially in the trauma of regions with a complex anatomy $[12,13]$. CT is not only used to better understand the fracture anatomy and make a surgical plan for both adult and child patients but also to investigate the occult pelvis, extremity, and vertebral fractures for which direct X-ray showed negative results $[4,14,15]$. It is often difficult to obtain a conventional radiographic series in trauma cases, and full-adaptation of patients to necessary positions cannot be achieved owing to pain. Clinicians should use a secondary imaging modality to rule out occult fractures in highly suspected patients with severe pain.

Patients with occult fractures may have a higher risk of complications $[14,16]$. In these patients, the lack of early diagnosis, and therefore, failure to initiate treatment may increase the complication rate. Immobilization is critical to shorten the healing time and prevent potential growth arrest, fracture deformity, and pain [14, 16]. However, the main limitation of this strategy is undesirable overtreatment, including unnecessary immobilization of extremity and follow-up period spanning 1-2 weeks. Early diagnosis facilitates a more effective treatment, a shorter hospital stay, and a long-term reduction in medical costs. Approximately half of the children without fractures were over-treated, and approximately one third of the children with fractures were under-treated [16]. In our study, 22 patients exhibited considerable number of fractures on MDCT, and these patients could have been under-treated using direct X-ray. The present study is important because our results demonstrated that the severity of pain in children with normal X-ray findings is indicative of the need for MDCT for detecting occult fractures. To the best of our knowledge, this is the first study to compare pain severity with MDCT results in pediatric trauma cases with normal X-ray findings.

Lesions on the tibia plateau, elbows, ankles, and wrists are often overlooked. Owing to their complex anatomy, the radiological evaluation of these sites may fail sometimes. Many studies have demonstrated the necessity of CT in the detection of occult fractures occurring in these regions $[17,18]$. These studies recommend that, in addition to patient history, physical examination, and direct X-ray, the mechanism of trauma should also be considered during initial examination at ED admission, and CT should be routinely used in case of clinical suspicion [19-22]. In another study, $19 \%$ of the fractures could not be diagnosed via standard X-ray procedure [23]. There is still a lack of consensus on the manner in which CT can be used for fracture detection. In an ED setting, VAS is the most commonly used pain scoring scale as well as the most suitable scale owing to its ease of application. Our study is important as it provides a methodology for deciding on the use of MDCT based on pain severity. The highest L-VAS scores were obtained for scaphoid, tibial plateau, talus, and calcaneus fractures, whereas the lowest L-VAS scores were obtained for intra-articular radial head fractures. In regions with such a complex anatomy, the possibility of fractures should be considered even if standard X-ray results are normal.

\section{Conclusion}

In conclusion, physicians should be alert regarding occult fractures and establish a systematic road map. We recommend the routine use of CT before performing conservative treatment despite normal X-ray results for pediatric patients with extremity trauma during initial examination at ED admission, especially if their pain severity is high.

\section{LIMITATIONS}

There are some limitations to this study. First, this was a single-center study, which was its main limitation, and second, when fractures were classified according to anatomical regions, there were less number of patients per group. Hence, multi-center prospective studies on this subject involving more cases are warranted.

\section{ACKNOWLEDGMENTS}

Thank numerous individuals participated in this study.

\section{CONFLICT OF INTEREST}

The Authors declares that there is no conflict of interest.

\section{REFERENCES}

[1] Spady DW, Saunders DL, Schopflocher DP, Svenson LW. Patterns of injury in children: a population-basedapproach. Pediatrics. 2004;113:522-9.

[2] Mathison JD, Agrawal D. General principles of fracture management: Fracture patterns and description in children. www.uptodate.com/home/index.html. Accessed June 14, 2010.

[3] Reisdorf E, Schwartz T. Introduction to emergency radiology. In Schwartz T. Reisdorf E, Editor. Emergency Radiology. 1 st ed. New York: McGraw Hill. 2000;p:1-10

[4] Freed HA, Shields NN. Most frequently overlooked radiographically apparent fractures in a teaching hospital emergency department. Ann Emerg Med. 1984;13:900-4.

[5] Jarraya M, Hayashi D, Roemer FW, Crema MD, Diaz L, Conlin J et al. Radiographically occult and subtle fractures: a pictorial review. Radiol Res Pract. 2013;2013:370169.

[6] Freyd M. The graphic rating scale. J Educ Psychol. 1923;14:83-102.

[7] Downie WW, Leatham PA, Rhind VW, Wright V, Branco JA, Anderson JA. Studies with pain rating scales. Ann Rheum Dis. 1978;37:378-81.

[8] Todd KH, Funk KG, Funk JP, Bonacci R. Clinical significance of reported changes in pain severity. Ann Emerg Med.1996;4:485-9.

[9] McGrath PJ1, Walco GA, Turk DC et al. Core outcome domains and measures for pediatric acute and chronic/recurrent pain clinical trials: PedIMMPACT recommendations. J Pain. 2008;9:771-83.

[10] Collins SL, Moore RA, McQuay HJ. The visual analogue pain 
intensity scale: what is moderate pain in millimetres? Pain. 1997;72:95-7.

[11] Buckwalter KA, Farber JM. Application of multidetector CT in skeletal trauma. Semin Musculoskelet Radiol. 2004;8:147-56.

[12] Pretorius ES, Fishman EK. Volume-rendered three-dimensional spiral CT: musculoskeletal applications. Radiographics. 1999;19:11431160 .

[13] Dalinka MK, Boorstein JM, Zlatkin MB. Computed tomography of musculoskeletal trauma. Radiol Clin North Am. 1989;27: 933-944.

[14] Naranja RJ Jr, Gregg JR, Dormans JP, Drummond DS, Davidson RS, Hahn M. Pediatric fracture without radiographic abnormality. Description and significance. Clin Orthop Relat Res. 1997;342: 1416.

[15] Allen GM, Wilson DJ, Bullock SA, Watson M. Extremity CT and ultrasound in the assessment of ankle injuries: occult fractures and ligament injuries. Br J Radiol. 2020;93:20180989.

[16] Berger PE, Ofstein RA, Jackson DW, Morrison DS, Silvino N, Amador R. MRI demonstration of radiographically occult fractures: what have we been missing? Radiographics. 1989;9: 407-36.

[17] Mallee W, Doornberg JN, Ring D, van Dijk CN, Maas M, Goslings JC. Comparison of CT and MRI for diagnosis of suspected scaphoid fractures. J Bone Joint Surg Am. 2011;5;93:20-28

[18] Memarsadeghi M, Breitenseher MJ, Schaefer-Prokop C, Weber M, Aldrian S, Gäbler C et al. Occult scaphoid fractures: comparison of multidetector CT and MR imaging-initial experience. Radiology. 2006;240:169-76.
[19] Holmes JF, Akkinepalli R. Computed tomography versus plain radiography to screen for cervical spine injury: a meta-analysis. $\mathrm{J}$ Trauma. 2005;58:902-5.

[20] Wei CJ, Tsai WC, Tiu CM, Wu HT, Chiou HJ, Chang CY. Systematic analysis of missed extremity fractures in emergency radiology. Acta Radiol. 2006;47:710-7.

[21] Welling RD, Jacobson JA, Jamadar DA, Chong S, Caoili EM, Jebson PJ. MDCT and radiography of wrist fractures: radiographic sensitivity and fracture patterns. AJR Am J Roentgenol. 2008;190:1016.

[22] Imerci A, Canbek U, Kaya A, Sürer L, Savran A. Distribution of occult fractures detected in emergency orthopedic patient trauma with computerized tomography. Ulus Travma Acil Cerrahi Derg. 2013;19:157-63

[23] Gandhi TK, Kachalia A, Thomas EJ, Puopolo AL, Yoon C, Brennan TA et al. Missed and delayed diagnoses in the ambulatory setting: a study of closed malpractice claims. Ann Intern Med. 2006;145:48896.

How to cite this article: Hüseyin Mutlu, Ahmet Çağlar, Ekrem Taha Sert. The Role of Pain Scoring in Multi-detector Computed Tomography Indications in Pediatric Occult. Signa Vitae. 2020;16(1):153-158. doi:10.22514/sv.2020.16.0020. 